\title{
Implications of Heart Rate in Patients with Left Ventricular Assist Devices
}

\author{
Teruhiko Imamura, ${ }^{1,2}$ MD, Koichiro Kinugawa, ${ }^{1}$ MD, Nikhil Narang, ${ }^{3}$ MD, Hidefumi Nishida, ${ }^{4}$ MD, \\ Sean Pinney, ${ }^{2} \mathrm{MD}$, Valluvan Jeevanandam, ${ }^{4} \mathrm{MD}$ and Takeyoshi Ota, ${ }^{4} \mathrm{MD}$
}

\begin{abstract}
Summary
Optimal heart rate (HR) is a promising therapeutic target in patients with heart failure with reduced ejection fraction. Nevertheless, the implication of optimal HR in patients with left ventricular assist devices (LVAD) remains unknown. The cohort included consecutive patients with sinus rhythm undergoing LVAD implantation between 2014 and 2018. Ideal HR was calculated as follows: $93-0.13 \times$ (deceleration time [msec]). The impact of "HR difference," defined as an HR difference between the actual HR at discharge and the calculated ideal HR, on the 1-year mortality and heart failure readmissions was investigated. A total of 143 patients (55 years old, $101 \mathrm{men}$ ) was identified and tertiled considering their HR differences: (1) the optimal HR group ( $n=$ 49; HR difference $<27 \mathrm{bpm})$, (2) the suboptimal HR group $(n=47$; HR difference $=27-42 \mathrm{bpm})$, and (3) the nonoptimal HR group; HR difference $(n=47$; HR difference $>43 \mathrm{bpm})$. The nonoptimal HR group had a significantly higher 1-year cumulative incidence of the primary endpoint compared with the optimal HR group (38\% versus $16 \%, P=0.029)$ with a hazard ratio of 1.69 (95\% confidence interval 1.02-2.57) adjusted for 6 potential confounders. In conclusion, nonoptimized HR negatively affected clinical outcomes in LVAD patients. The implication of deceleration time-guided HR optimization in LVAD patients should be further investigated.
\end{abstract}

Key words: Echocardiography, Deceleration time, Ivabradine

(Int Heart J 2022; 63: 56-61)

$\mathrm{M}$ anagement of patients with continuous-flow left ventricular assist devices (LVADs) has evolved positively with the new iterations of available devices along with coinciding with improved clinical outcomes. ${ }^{1)}$ Nevertheless, morbidity following LVAD support remains an ongoing challenge. ${ }^{2}$

The implication of heart rate (HR) modulation is receiving great concern given the recent introduction of ivabradine, which is a selective inhibitor of $I_{f}$ channels that exclusively reduces HR and improves prognosis in heart failure patients with reduced ejection fraction and sinus rhythm as demonstrated in the SHIFT trial. ${ }^{3)}$ However, the optimal target HR range to maximize benefit remains controversial. ${ }^{4)}$ Furthermore, whether these target HR ranges can be widely extrapolated to several clinical cohorts is unknown.

Our group recently proposed a formula to calculate the ideal HR for each individual with systolic dysfunction using the deceleration time of E-wave obtained from transmitral Doppler echocardiography. ${ }^{5)}$ At the ideal HR, E-wave and A-wave stand adjacent to each other and the cardiac output is theoretically maximized. ${ }^{6}$ A maximized filling in the left ventricle might minimize cardiac potential energy per minute and facilitate cardiac reverse remodeling even in patients with LVAD supports.
We hypothesized that optimal HR within the range of the calculated ideal HR would be associated with improved clinical outcomes along with a higher odds of cardiac reverse remodeling even in LVAD patients.

\section{Methods}

Patient selection: Consecutive patients who underwent LVAD implantation at the University of Chicago Medical Center between 2014 and 2018 were reviewed. Patients who died during the index hospitalization, had atrial fibrillation, had no available echocardiographic studies at the index discharge, had implanted a pacemaker with underlying pacing, and/or were followed for less than 1 year after the index discharge were excluded. In principle, all patients had scheduled clinic visits once per month. This study was approved by the local institutional review board.

HR assessment (ideal HR versus actual HR): The actual HR at rest was measured at the time of index discharge using an electrocardiogram, which was performed after a 10-minute rest period in the supine position. Transthoracic echocardiography was performed simultaneously and the deceleration time of E-wave in the transmitral Doppler echocardiography was measured.

From the ${ }^{1}$ Second Department of Internal Medicine, University of Toyama, Toyama, Japan, ${ }^{2}$ Department of Medicine, University of Chicago Medical Center, Chicago, USA, ${ }^{3}$ Advocate Christ Medical Center, Oak Lawn, USA and ${ }^{4}$ Department of Surgery, University of Chicago Medical Center, Chicago, USA.

Address for correspondence: Takeyoshi Ota, MD, Department of Surgery, The University of Chicago, 5841 S Maryland Avenue, MC5040, Chicago, IL 60637, USA. E-mail: tota@bsd.uchicago.edu

Received for publication July 23, 2021. Revised and accepted November 6, 2021.

doi: $10.1536 /$ ihj.21-468

All rights reserved by the International Heart Journal Association. 
A

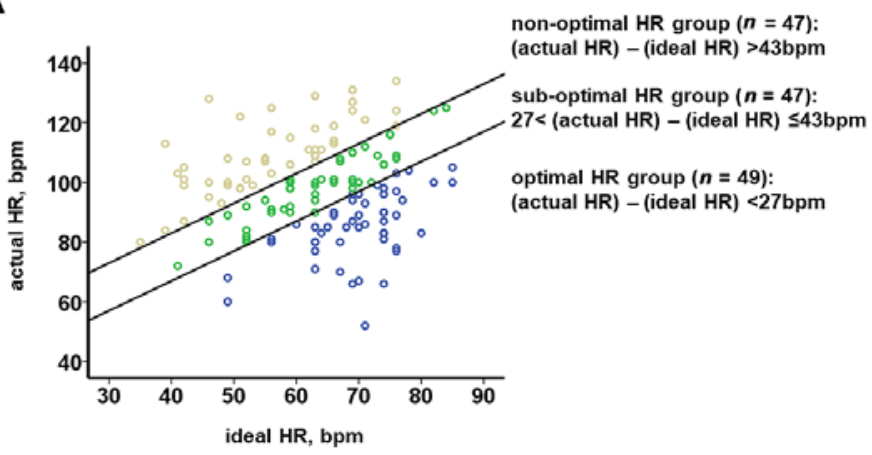

B

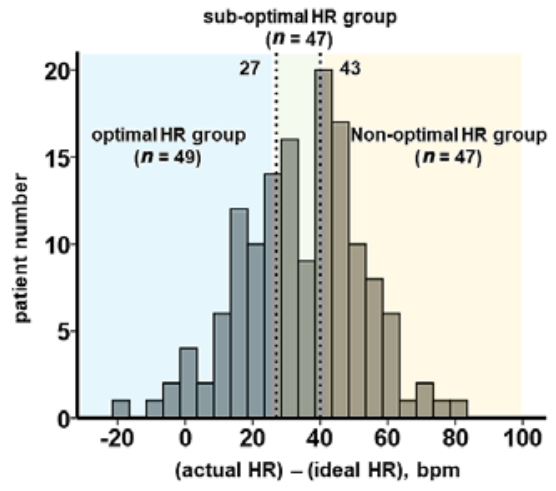

Figure 1. Correlation between ideal HR and actual HR (A); distribution of the difference between actual HR and ideal HR (B). Black lines indicate cutoffs of HR difference, i.e., $27 \mathrm{bpm}$ and $43 \mathrm{bpm}$. For example, when a patient has $100 \mathrm{bpm}$ of actual HR and $45 \mathrm{bpm}$ of ideal HR, the HR difference is calculated as $55 \mathrm{bpm}$ ( $>47 \mathrm{bpm}$ ). The patient is assigned to the nonoptimal HR group (gray dot).

The ideal HR was calculated according to the following formula: $93-0.13 \times($ deceleration time $[\mathrm{msec}]){ }^{5)} \mathrm{A}$ difference between actual HR and ideal HR was calculated for each patient. All patients were tertiled on the basis of the HR difference.

Data collection: The primary endpoint was a composite of 1-year all-cause death and readmissions due to heart failure that was defined as volume overload requiring inhospital IV diuretic therapy. Heart transplantation was censored. Demographic, laboratory, medication, and echocardiographic data were obtained at the time of index discharge (day 0). Echocardiography was repeated 1 year from the LVAD implantation. Data on medications were obtained also at a 1-year follow-up.

Transthoracic echocardiography was performed by expert sonographers blinded to the study protocol. Valvular regurgitations were graded as none/trace, mild, mildmoderate, moderate, moderate-severe, and severe. Right ventricular size and dysfunction were graded as normal, mild, mid-moderate, moderate, and severe. Grades of moderate or greater were defined as significant.

Statistical analyses: Primary outcomes were compared among the groups tertiled by the HR difference. Statistical analyses were performed using SPSS Statistics 22 (SPSS Inc, Armonk, IL, USA). Two-sided $P$-values of $<0.05$ were considered statistically significant.

Continuous variables were expressed as median $(25 \%$ interquartile, $75 \%$ interquartile) and compared using the Kruskal-Wallis test. Categorical variables were expressed as numbers and percentages and compared using Fisher's exact test. The trend of continuous variables between the index discharge and 1-year follow-up was assessed using the Wilcoxon signed-rank test. The trend of categorical variables was assessed using the McNemar test.

Cumulative incidences of the primary endpoint were compared among the groups using a log-rank test. Association of HR difference on the primary endpoint was adjusted for clinically significant variables including age, body surface area, destination therapy, the use of betablockers, left ventricular end-diastolic diameter, and tricuspid annular plane systolic excursion using Cox proportional hazard ratio regression analysis. Heart failure readmission rates per year were compared among the groups using negative binomial regression analyses.

\section{Results}

Baseline characteristics: A total of 204 patients underwent LVAD implantation. Among them, 61 patients were excluded on the basis of the aforementioned exclusion criteria and 143 patients were included in the cohort. The median age was $55(47,66)$ years, and $101(71 \%)$ patients were males. The majority $(76 \%)$ received LVAD implantation as destination therapy. No patients received ivabradine or antiarrhythmics.

Stratification according to the HR difference: The actual HR obtained at index discharge was $99(87,108)$ bpm. Given the measured deceleration time was 228 (167, 286) msec, ideal HR was calculated as $63(55,71) \mathrm{bpm}$. The difference between actual and ideal HR was 35 (22, 47) bpm. Figure 1A shows the distribution of actual and ideal HR.

Patients were tertiled according to the difference between actual HR and ideal HR at cutoffs of 27 and 43 bpm: (1) the optimal HR group ( $\leq 27 \mathrm{bpm}, n=49$ ), (2) the suboptimal HR group ( $>27$ and $\leq 43$ bpm, $n=47$ ), and (3) the nonoptimal HR group ( $>43 \mathrm{bpm}, n=47$ ) (Figure 1B).

Comparison of baseline characteristics: There were no statistically significant differences in the demographics and comorbidities between the groups $(P>0.05$ for all; Table I). Deceleration time was shorter and ideal HR was higher in the optimal HR group. Actual HR was lower in the optimal HR group.

Trend in medications: At index discharge, the prevalence of medications utilized was not statistically different between the groups (Table II). At 1-year follow-up, only the prevalence of beta-blocker use was significantly increased in the nonoptimal HR group $(P=0.001)$. The prevalence of all medications was not significantly different among the groups at 1 -year follow-up $(P>0.05$ for all).

Trend in echocardiographic parameters: Table III summarizes the trend in echocardiographic parameters. Echocardiographic data at index discharge did not significantly differ between the groups $(P>0.05$ for all). At 1-year follow-up, left ventricular end-diastolic diameter signifi- 
Table I. Baseline Characteristics at Index Discharge

\begin{tabular}{|c|c|c|c|c|c|}
\hline & Total $(n=143)$ & Optimal $(n=49)$ & Suboptimal $(n=47)$ & Abnormal $(n=47)$ & $P$ value \\
\hline \multicolumn{6}{|l|}{ Demographics } \\
\hline Age, years & $55(47,66)$ & $58(50,66)$ & $52(47,65)$ & $53(42,69)$ & 0.23 \\
\hline Male sex & $101(71 \%)$ & $36(73 \%)$ & $33(70 \%)$ & $32(68 \%)$ & 0.84 \\
\hline Body surface area, $\mathrm{m}^{2}$ & $2.11(1.92,2.30)$ & $2.07(1.91,2.23)$ & $2.16(1.99,2.31)$ & $2.08(1.87,2.33)$ & 0.25 \\
\hline Ischemic etiology & $41(29 \%)$ & $18(37 \%)$ & $11(23 \%)$ & $12(26 \%)$ & 0.30 \\
\hline Destination therapy & $108(76 \%)$ & $41(84 \%)$ & $33(70 \%)$ & $34(72 \%)$ & 0.36 \\
\hline $\begin{array}{l}\text { Length of hospitalization follow- } \\
\text { ing surgery, days }\end{array}$ & $20(15,28)$ & $19(16,29)$ & $22(16,28)$ & $23(16,33)$ & 0.24 \\
\hline Device type & & & & & 0.13 \\
\hline HeartMate II & $79(55 \%)$ & $29(59 \%)$ & $28(60 \%)$ & $22(47 \%)$ & - \\
\hline HeartWare & $30(21 \%)$ & $13(27 \%)$ & $6(13 \%)$ & $11(23 \%)$ & - \\
\hline HeartMate 3 & $34(24 \%)$ & $7(14 \%)$ & $13(28 \%)$ & $14(30 \%)$ & - \\
\hline INTERMACS profile & & & & & 0.52 \\
\hline Profile 2 & $12(8 \%)$ & $4(8 \%)$ & $5(11 \%)$ & $3(6 \%)$ & - \\
\hline Profile 3 & $56(39 \%)$ & $15(31 \%)$ & $21(45 \%)$ & $20(43 \%)$ & - \\
\hline Profile 4-7 & $75(52 \%)$ & $30(61 \%)$ & $21(45 \%)$ & $24(51 \%)$ & - \\
\hline \multicolumn{6}{|l|}{ Comorbidities } \\
\hline Diabetes mellitus & $49(34 \%)$ & $17(35 \%)$ & $13(28 \%)$ & $19(40 \%)$ & 0.43 \\
\hline History of stroke & $23(16 \%)$ & $7(14 \%)$ & $8(17 \%)$ & $8(17 \%)$ & 0.92 \\
\hline $\begin{array}{l}\text { History of ventricular tachyar- } \\
\text { rhythmia }\end{array}$ & $52(36 \%)$ & $18(37 \%)$ & $16(34 \%)$ & $18(38 \%)$ & 0.91 \\
\hline Chronic kidney disease & $47(33 \%)$ & $14(29 \%)$ & $17(36 \%)$ & $16(34 \%)$ & 0.72 \\
\hline $\begin{array}{l}\text { Chronic obstructive pulmonary } \\
\text { disease }\end{array}$ & $29(20 \%)$ & $10(20 \%)$ & $11(23 \%)$ & $8(17 \%)$ & 0.74 \\
\hline Atrial fibrillation & 0 & 0 & 0 & 0 & 1.0 \\
\hline \multicolumn{6}{|l|}{ Laboratory parameters } \\
\hline Hemoglobin, g/dL & $9.2(8.3,10.5)$ & $9.1(8.4,10.7)$ & $9.4(8.5,11.0)$ & $9.0(8.2,10.4)$ & 0.31 \\
\hline Serum total bilirubin level, $\mathrm{mg} / \mathrm{dL}$ & $0.9(0.7,1.2)$ & $0.9(0.7,1.2)$ & $0.8(0.7,1.3)$ & $0.9(0.7,1.2)$ & 0.65 \\
\hline eGFR, $\mathrm{mL} /$ minute $/ 1.73 \mathrm{~m}^{2}$ & $55.4(46.2,68.7)$ & $54.1(45.1,67.3)$ & $57.1(47.2,69.2)$ & $56.8(48.1,70.2)$ & 0.58 \\
\hline \multicolumn{6}{|l|}{ Heart rate parameter } \\
\hline E-wave height, $\mathrm{cm} / \mathrm{sec}$ & $84(59,104)$ & $85(64,104)$ & $83(58,107)$ & $88(65,108)$ & 0.65 \\
\hline Deceleration time, msec & $228(167,286)$ & $174(144,208)$ & $234(182,277)$ & $286(231,342)$ & $<0.001 *$ \\
\hline Actual heart rate, bpm & $99(87,108)$ & $85(80,94)$ & $100(91,106)$ & $109(101,119)$ & $<0.001 *$ \\
\hline Ideal heart rate, bpm & $63(55,71)$ & $70(66,74)$ & $63(56,69)$ & $56(48,63)$ & $<0.001^{*}$ \\
\hline $\begin{array}{l}\text { Difference between actual and } \\
\text { ideal heart rate, bpm }\end{array}$ & $35(22,47)$ & $17(10,23)$ & $35(32,41)$ & $51(47,58)$ & $<0.001 *$ \\
\hline
\end{tabular}

Continuous variables were expressed as median and interquartile and compared among the groups using the Kruskal-Wallis test. Categorical variables were expressed as numbers and percentages and compared among the groups using Fisher's exact test. $* P<0.05$. eGFR indicates the estimated glomerular filtration ratio.

cantly decreased and the prevalence of moderate or greater right ventricular dysfunction and right ventricular size significantly decreased in the optimal HR group $(P<$ 0.05 for both). More patients had increases/worsening in ventricular sizes and right atrial size, valvular regurgitation, and right heart function in the nonoptimal HR group. Death or heart failure readmission: During the 1-year observational period, 17 patients died (due to 5 stroke, 4 pump thrombosis, 3 sepsis, 1 heart failure, and 4 unknown origin) and 23 patients experienced heart failure readmissions. The cumulative incidences of primary endpoints were stratified into 3 groups: optimal HR group $16 \%$, suboptimal HR group 24\%, and nonoptimal HR group 38\% ( $P=0.029$; Figure $2 \mathrm{~A})$.

The hazard ratio for the primary endpoint, which was adjusted for 6 clinically significant variables were 1.47 (95\% confidence interval $0.58-3.78, P=0.32$ ) in the suboptimal HR group and 1.69 (95\% confidence interval 1.02-2.57, $P=0.014$ ) in the nonoptimal HR group, in comparison with the optimal HR group as a reference.
The primary endpoint did not demonstrate a significant association when considering the actual HR as a continuous variable (hazard ratio 1.01 [95\% confidence interval $0.99-1.03$ ], $P=0.41$ ) and a tertiled categorical variable (hazard ratio 1.02 [95\% confidence interval 0.68 1.52], $P=0.93$ ).

Heart failure readmission rate: During the study period, there were 33 heart failure readmissions in 23 patients. Heart failure readmission rates were 0.400 events per year for the nonoptimal HR group, 0.207 events per year for the suboptimal HR group, and 0.153 events per year for the optimal HR group (Figure 2B).

\section{Discussion}

In this study, we investigated the impact of optimal HR on the risk of mortality and heart failure readmissions following LVAD implantation. The main findings are as follows: (1) Most patients had higher HR at index discharge compared with the ideal HR. (2) The size of the 
Table II. Clinical Data at Index Discharge and 1 Year Later

\begin{tabular}{|c|c|c|c|c|c|c|c|}
\hline & $\begin{array}{l}\text { Optimal } \\
(n=49)\end{array}$ & & $\begin{array}{l}\text { Suboptimal } \\
\quad(n=47)\end{array}$ & & $\begin{array}{c}\text { Abnormal } \\
(n=47)\end{array}$ & & $\begin{array}{c}\text { Intergroup } \\
P \text { value }\end{array}$ \\
\hline & & $\begin{array}{c}\text { Intragroup } \\
P \text { value }\end{array}$ & & $\begin{array}{c}\text { Intragroup } \\
P \text { value }\end{array}$ & & $\begin{array}{c}\text { Intragroup } \\
P \text { value }\end{array}$ & \\
\hline \multicolumn{8}{|l|}{ At index discharge } \\
\hline Beta-blocker & $23 / 49(47 \%)$ & - & $24 / 45(53 \%)$ & - & $16 / 45(36 \%)$ & - & 0.23 \\
\hline ACEI/ARB/ARNI & $29 / 49(59 \%)$ & - & $21 / 45(47 \%)$ & - & $22 / 45(49 \%)$ & - & 0.43 \\
\hline MRA & $30 / 49(61 \%)$ & - & $19 / 45(42 \%)$ & - & $20 / 45(44 \%)$ & - & 0.13 \\
\hline Diuretics & $43 / 49(88 \%)$ & - & $36 / 45(80 \%)$ & - & $33 / 45(75 \%)$ & - & 0.21 \\
\hline Intravenous inotropes & $0 / 49(0 \%)$ & - & $1 / 45(2 \%)$ & - & $0 / 45(0 \%)$ & - & 0.56 \\
\hline Device flow, L/minute & $4.2(3.1,5.1)$ & - & $4.4(3.0,5.2)$ & - & $4.3(3.5,5.4)$ & - & 0.75 \\
\hline Body surface area, $\mathrm{m}^{2}$ & $2.07(1.91,2.23)$ & - & $2.16(1.99,2.31)$ & - & $2.08(1.87,2.33)$ & - & 0.25 \\
\hline \multicolumn{8}{|l|}{ One year later } \\
\hline Beta-blocker & $25 / 40(63 \%)$ & 0.18 & $28 / 41(68 \%)$ & 0.21 & $29 / 39(74 \%)$ & $0.001 *$ & 0.53 \\
\hline ACEI/ARB/ARNI & $24 / 40(60 \%)$ & 1.0 & $17 / 41(41 \%)$ & 0.087 & $23 / 39(59 \%)$ & 0.076 & 0.56 \\
\hline MRA & $18 / 40(45 \%)$ & 0.092 & $13 / 41(32 \%)$ & 0.39 & $16 / 39(41 \%)$ & 0.27 & 0.45 \\
\hline Diuretics & $24 / 40(60 \%)$ & 1.0 & $31 / 41(76 \%)$ & 1.0 & $28 / 39(72 \%)$ & 1.0 & 0.22 \\
\hline Intravenous inotropes & $0 / 40(0 \%)$ & - & $1 / 41(2 \%)$ & 1.0 & $0 / 39(0 \%)$ & - & 0.59 \\
\hline Device flow, L/minute & $4.2(3.3,4.6)$ & 0.25 & $4.3(3.4,5.3)$ & 0.053 & $4.1(3.4,4.9)$ & 0.11 & 0.58 \\
\hline Bosy surface area, $\mathrm{m}^{2}$ & $2.03(1.87,2.18)$ & 0.36 & $2.12(1.96,2.28)$ & 0.26 & $2.15(1.93,2.41)$ & 0.087 & 0.24 \\
\hline
\end{tabular}

ACEI indicates angiotensin-converting enzyme inhibitor; ARB, angiotensin II receptor blocker; ARNI, angiotensin receptor neprilysin inhibitor; and MRA, mineralocorticoid receptor antagonist. Continuous variables were expressed as median interquartile. The intergroup comparison was performed using the Kruskal-Wallis test. The trend of variables between the index discharge and 1 year later was assessed using the Wilcoxon signed-rank test. Categorical variables were expressed as numbers. The intergroup comparison was performed using Fisher's exact test. The trend of variables between the index discharge and 1 year later (i.e., intragroup comparison) was assessed using the McNemar test.

left ventricle decreased, and right ventricular function improved in the optimal HR group, whereas they remained unchanged in other groups. (3) Nonoptimal HR (i.e., significantly higher HR over the ideal HR) was associated with a higher cumulative incidence of death or heart failure readmission.

Ideal HR: With an ideal HR range, at which the overlap length between E-wave and A-wave in the transmitral Doppler echocardiographic flow is "zero," left ventricular filling is theoretically maximized and cardiac potential energy per minute is minimized. ${ }^{6}$ This would potentially allow for cardiac reverse remodeling. Although an ideal HR may vary between patients, this concept sets up the framework for an individualized HR target range, instead of an absolute and fixed HR target. ${ }^{5}$

Inappropriate tachycardia is associated with increased myocardial oxygen demand and high potential energy expenditure in patients with left ventricular systolic dysfunction. Given the promising impact of beta-blocker therapy in improving hemodynamics and facilitating reverse remodeling in LVAD patients, appropriate HR modulation might reduce potential energy and improve diastolic filling. ${ }^{7}$ Furthermore, higher HR decreases diastolic filling, ${ }^{6}$ which is required to maintain preload also in LVAD patients. Applying the benefits of chronotropic modulation of beta-blockade in HFrEF patients, a lower HR might be beneficial in reverse remodeling also in LVAD patients. ${ }^{7}$

By contrast, severe bradycardia is not physiologically beneficial as diastolic filling falls despite a minimized myocardial oxygen demand. Systemic flow is largely dependent on the preload during LVAD support, and an adequate left ventricular filling is essential to maintain sufficient systemic flow regarding the unique physiology of LVADs. The extremes of bradycardia may lead to im- paired diastolic filling. Compensatory sympathetic mechanisms to this may also worsen the physiological milieu needed for cardiac reverse remodeling during LVAD support.

Cardiac reverse remodeling and heart failure readmission: We observed that left ventricular dimensions decreased at 1 year following LVAD implantation only in the optimal HR group. The prevalence of medication including beta-blocker did not differ among the groups. We did not assess left ventricular ejection fraction as a surrogate given the presence of a continuous-flow device. For the overall cohort, the right ventricular function also improved only in the optimal HR group, probably because of improved afterload on the right ventricle. Moreover, nonoptimal HR was associated with a higher incidence of mortality or heart failure readmission after adjustment for baseline clinically relevant covariates.

A subanalysis of the SHIFT trial demonstrated that the HR reduction using ivabradine was associated with an improved cardiac function compared with the placebo arm. ${ }^{8)}$ Such an improvement in cardiac remodeling, which was defined as left ventricular end-systolic volume index $<59 \mathrm{~mL} / \mathrm{m}^{2}$, was associated with higher freedom from cardiovascular death or heart failure readmission.

Medical therapy to optimize HR: Further prospective studies in the mechanical circulatory support population are needed to better validate the potential benefit of ivabradine. Although true myocardial recovery following LVAD implantation remains relatively uncommon, a tailored protocol of introducing guideline-directed therapies to optimize HR and blood pressure may offer the best chance of recovery amenable to explant devices in selected patients. ${ }^{9}$

Future perspective: Abnormal hemodynamics, particu- 
Table III. Transthoracic Echocardiography at Index Discharge and 1 Year Later

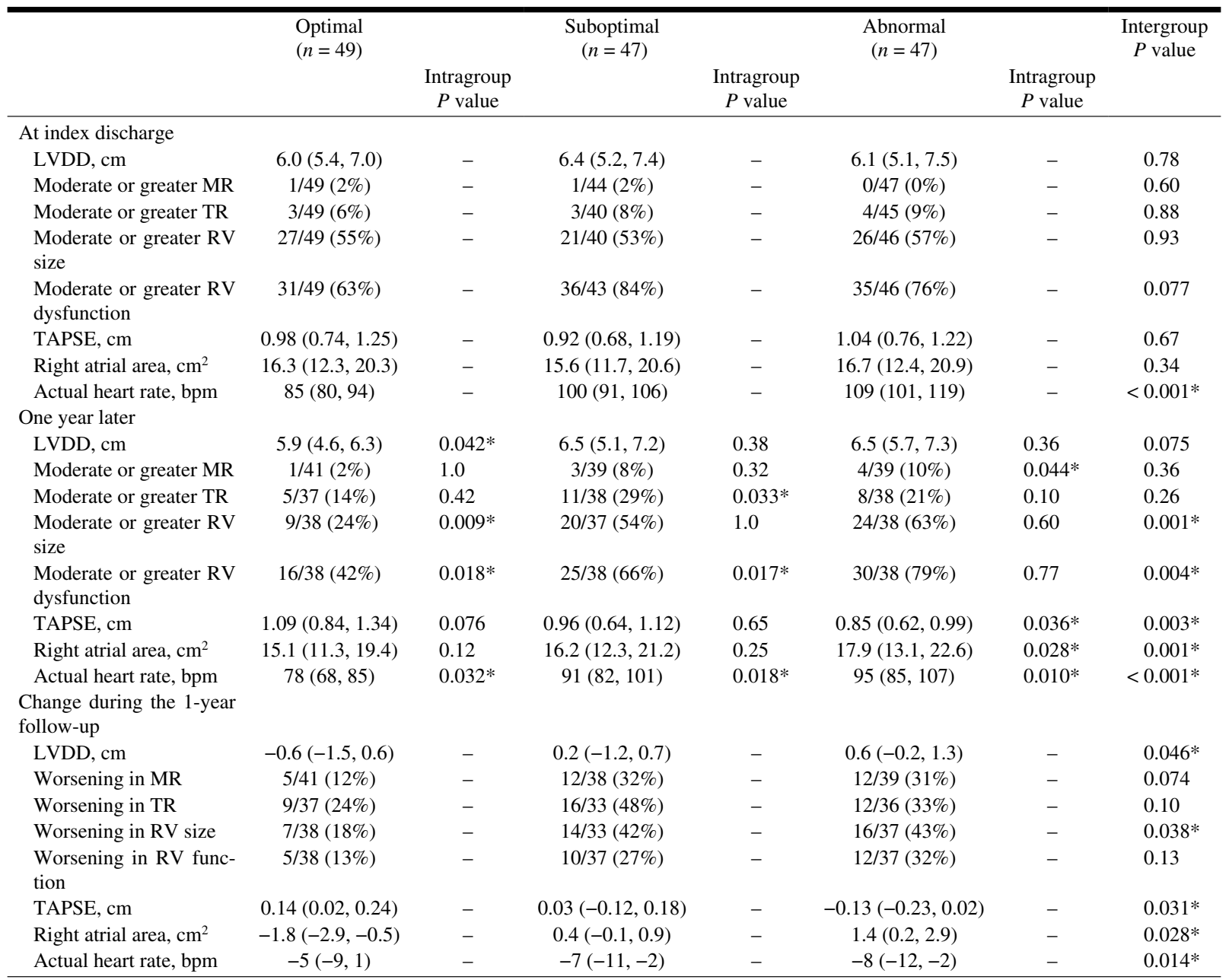

LVDD indicates left ventricular end-diastolic diameter; MR, mitral regurgitation; TR, tricuspid regurgitation; RV, right ventricle; and TAPSE, tricuspid annular plane systolic excursion. Continuous variables were expressed as median interquartile. The intergroup comparison was performed using the Kruskal-Wallis test. The trend of variables between the index discharge and 1 year later was assessed using the Wilcoxon signed-rank test. Categorical variables were expressed as numbers. The intergroup comparison was performed using Fisher's exact test. The trend of variables between the index discharge and 1 year later (i.e., intragroup comparison) was assessed using the McNemar test. $* P<0.05$.

larly elevated central venous pressures indicative of right heart dysfunction, are associated with hemocompatibilityrelated adverse events including gastrointestinal bleeding, stroke, and pump thrombosis via the activation of inflammatory and angiogenesis cascades. ${ }^{10)}$ The association between nonoptimal HR and comorbidities other than heart failure should be examined.

The present study lacks comprehensive hemodynamic data. Such data would help better clarify how and why optimal HR was associated with cardiac reverse remodeling and prevention of heart failure recurrence.

Limitations: First, this study is a proof of concept and included only a limited sample size. We did adjust clinically significant confounders, but other uninvestigated factors might have a considerable impact on the outcomes. Second, we tertiled patients considering the difference between actual HR and ideal HR: the optimal HR, suboptimal HR, and nonoptimal HR groups. More optimal cutoffs that better stratify the clinical outcomes might exist.
Third, this study is based on the concept that a deceleration time is constant in each individual. ${ }^{5)}$ This assumption is reasonable when clinical conditions remain stable, although in itself must be further investigated. Fourth, we did not consider the trend of HR, which may also act as a risk modifier. Fifth, there were no patients with actual HR significantly below the ideal HR. The implication of low HR below the ideal range remains uninvestigated. Sixth, we hypothesized that the predominant determinants of mitral inflow overlap length were HR and deceleration time; other parameters including systolic duration and E-wave duration might also have considerable impacts, which were not evaluated in this study. Seventh, we excluded those with atrial fibrillation or depending on the pacemaker, given a different prognostic impact of their HR.

\section{Conclusion}

Nonoptimized HR negatively affected clinical out- 

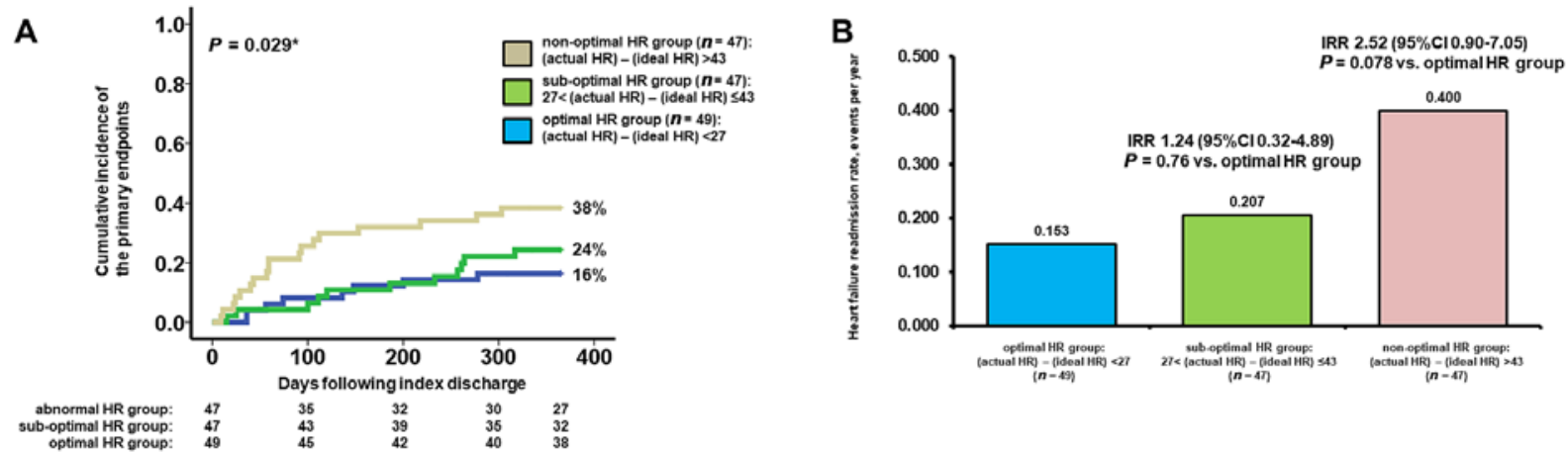

Figure 2. Cumulative incidence of death or heart failure readmission $(\mathbf{A})$ and heart failure readmission rate per year $(\mathbf{B})$ stratified by the tertiled HR difference. Cumulative incidences were compared using a log-rank test. Event rates were compared using negative binomial regression analysis. $* P<0.05$.

comes in LVAD patients. The implication of deceleration time-guided HR optimization in LVAD patients needs further investigation.

\section{Disclosure}

Conflicts of interest: TI receives grant support from JSPS KAKENHI: JP20K17143. VJ is a consultant for Abbott Inc., Medtronic Inc., and Reliant Heart Inc. SP is a consultant for Abbott Inc., Medtronic Inc.

\section{References}

1. Teuteberg JJ, Cleveland JC, Jr., Cowger J, et al. The Society of Thoracic Surgeons intermacs 2019 annual report: the changing landscape of devices and indications. Ann Thorac Surg 2020; 109: 649-60.

2. Hasin T, Marmor Y, Kremers W, et al. Readmissions after implantation of axial flow left ventricular assist device. J Am Coll Cardiol 2013; 61: 153-63.

3. Swedberg K, Komajda M, Böhm M, et al. Ivabradine and outcomes in chronic heart failure (SHIFT): a randomised placebocontrolled study. Lancet 2010; 376: 875-85.
4. Böhm M, Swedberg K, Komajda M, et al. Heart rate as a risk factor in chronic heart failure (SHIFT): the association between heart rate and outcomes in a randomised placebo-controlled trial. Lancet 2010; 376: 886-94.

5. Izumida T, Imamura T, Nakamura M, Fukuda N, Kinugawa K. How to consider target heart rate in patients with systolic heart failure. ESC Heart Fail 2020; 7: 3231-4.

6. Hori M, Imamura T, Narang N, Kinugawa K. Implications of doppler echocardiography-guided heart rate modelation using ivabradine. Intern Med 2021; 7343-21.

7. Yousefzai R, Brambatti M, Tran HA, et al. Benefits of neurohormonal therapy in patients with continuous-flow left ventricular assist devices. ASAIO J 2020; 66: 409-14.

8. Tardif JC, O'Meara E, Komajda M, et al. Effects of selective heart rate reduction with ivabradine on left ventricular remodelling and function: results from the SHIFT echocardiography substudy. Eur Heart J 2011; 32: 2507-15.

9. Birks EJ, Drakos SG, Patel SR, et al. A prospective multicentre study of myocardial recovery using left ventricular assist devices (REmission from Stage D heart failure: RESTAGE-HF): medium term and primary endpoint results. Circulation 2020; 142: $2016-28$

10. Imamura T, Nguyen A, Kim G, et al. Optimal haemodynamics during left ventricular assist device support are associated with reduced haemocompatibility-related adverse events. Eur J Heart Fail 2019; 21: 655-62. 